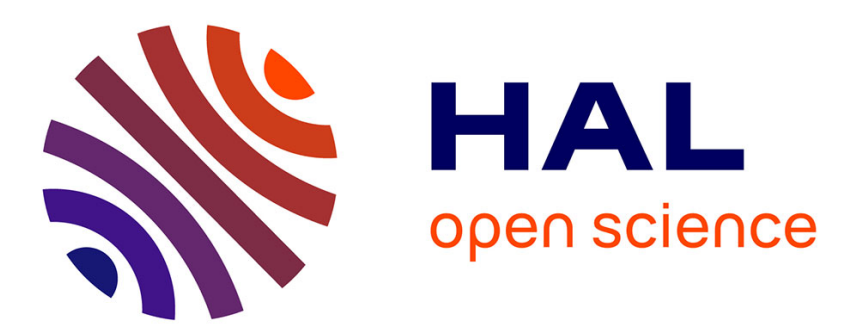

\title{
Classic and Adaptive Washout Comparison for a Low Cost Driving Simulator
}

Lamri Nehaoua, Ali Amouri, Hichem Arioui

\section{To cite this version:}

Lamri Nehaoua, Ali Amouri, Hichem Arioui. Classic and Adaptive Washout Comparison for a Low Cost Driving Simulator. 13th IEEE Mediterranean Conference on Control and Automation (MED 2005), Jun 2005, Limassol, Cyprus. pp.586-591, 10.1109/.2005.1467080 . hal-00342962

\section{HAL Id: hal-00342962 \\ https://hal.science/hal-00342962}

Submitted on 29 May 2012

HAL is a multi-disciplinary open access archive for the deposit and dissemination of scientific research documents, whether they are published or not. The documents may come from teaching and research institutions in France or abroad, or from public or private research centers.
L'archive ouverte pluridisciplinaire HAL, est destinée au dépôt et à la diffusion de documents scientifiques de niveau recherche, publiés ou non, émanant des établissements d'enseignement et de recherche français ou étrangers, des laboratoires publics ou privés. 


\title{
Classic and Adaptive Washout Comparison for a Low Cost Driving Simulator
}

\author{
L. Nehaoua ${ }^{1}$, A. Amouri ${ }^{2}$ and H.Arioui ${ }^{2}$
}

\begin{abstract}
- the paper deals with control of small driving simulator $\mathrm{SIM}^{2}$ comparing the useless of adaptive and classic approaches. Driving simulators are considering as an interactive virtual reality tools, which take a considerable place in the human factors studies. The difficulty to reproduce in reality some drive situations mainly for risk and reproducibility reasons increases the interest of this tool. Nevertheless, the validation of the experiments carried out on driving simulator is closely related to embedding realism of the driver in the simulated world.

In this article, we present the design of a low cost and small motion platform, which allow the restitution of 2 DOF movements. This overall system is considered as two independent systems linked mechanically. The first system consists in motorized rail for the longitudinal movement while the second system consists in motorized seat allowing either pitch movement of this one or just back seat inclination (this case will not be discussed in the rest of paper).
\end{abstract}

\section{INTRODUCTION}

Some tasks, which are easily achieved in an actual driving situation (lane shift or queuing), become tedious when the driver has to accomplish them using a driving simulator, primarily when it is a static one. The lacks of sensory stimuli (like haptic one) prevent the driver from an adequate control of the virtual vehicle. In order to drive a virtual vehicle, the driver needs to be provided with sufficient information which allows him to control the car as easily as it is the case in most of real situations.

Depending on the hardware architecture of each simulator, the feedback strategies might be different, due to the very fact that the control is based on sensory-motor activity.

Some studies aiming to highlight the relevance of kinesthetic perception in simulator controllability clearly showed that longitudinal and lateral accelerations significantly reduce the simulator control variability [1,2,3].

Accurate restitution of accelerations observed on an actual vehicle is impossible to achieve on a driving simulator, whatever motion platform performances are (as acceleration restitution during a braking maneuver can

\footnotetext{
${ }^{1}$ CIR-INRETS, 2 Avenue du Général Malleret - Joinville. F-94114 Arcueil Cedex, France (e-mail : nehaoua@inrets.fr).

${ }^{2}$ LSC-CNRS Fre 2494. 40, Rue du Pelvoux, 91025 Courcouronnes, France (e-mail : arioui, amouri@iup.univ-evry.fr).
}

demand a platform shift of around 100 meters) [4]. Due to this impossibility, illusion of inertial effect has to be provided to the driver. Such illusion rests on acquired knowledge of the human perceptive system. In the case of continuous accelerations the illusion is generally produced by tilting the driver forward or backward. Such tilt can be interpreted by his/her vestibular system, as either a positive or negative acceleration, depending on the direction of the tilt $[3,5]$. In the transient acceleration case, the platform is linearly moved in the same acceleration direction and come back when the acceleration is continuous. The implementation of this technique depends strongly on the architecture of the motion platform, the limits of its workspace and its bandwidth capacity as well as on the dynamic characteristics of the actuators used to move the platform [6,7].

The first designed motion platforms were intended for movement restitution for flight simulators. The concept was applied much later to movement restitution on automobile driving simulators. The designers of the first driving simulators which, integrated motion platforms derived from technology used for flight simulators, were confronted with a several problems linked to the differences between driving a car and flying a plane as:

The dynamics of a vehicle are indeed different from those of an airplane; and the 6 DOF acceleration variations in a vehicle are more frequent and sometimes more brutal than those observed in airplane (in particular in bends, when changing lanes or braking).

Driving a vehicle takes place in traffic that can sometimes create very complex situations. The driver is thus more called upon for the control of his vehicle than is an airplane pilot (handling interactions and, notably, car following driving).

The sensory information used for driving a vehicle are greater (and sometimes different) that the ones used for flying an airplane.

All these constraints have led designers to imagine another architecture by seeking as often as possible to supply the driver with stimuli that are as close as possible to those existing in actual situations.

To do this, we have designed and built a low-cost motion platform equipped with two degrees of freedom as shown in figure(1) [6]. This makes it possible to animate the 
simulator's cab with a longitudinal movement, on the one hand, and with a weak rotation movement from the driver's seat or a weak tilt of the back of this seat, on the other hand.

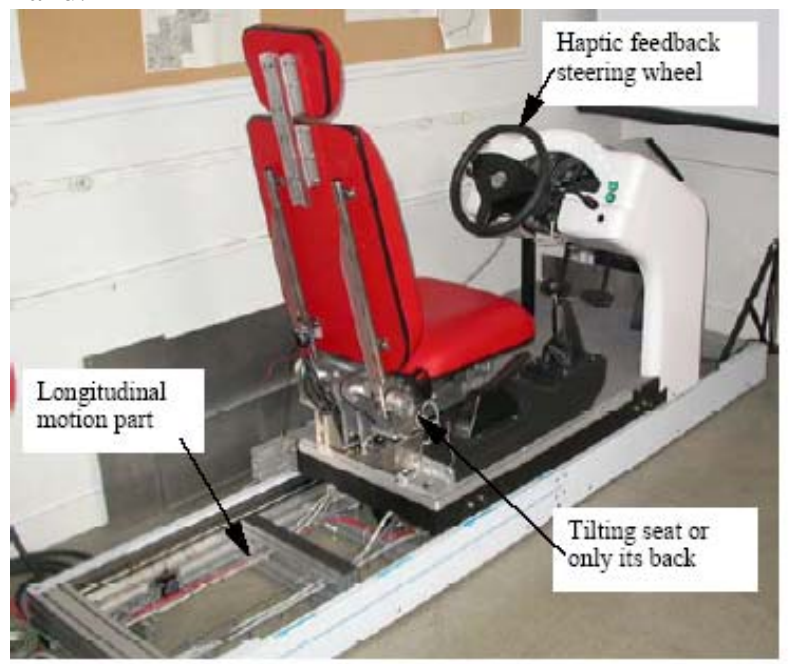

Figure 1: platform architecture

\section{PlATFORM MODELLING}

To model the driving simulator motion, the overall system is considered as two independent systems mechanically linked: the rotating driving seat and the longitudinal motion platform. Each of them is driven by a single actuator. The motion platform undergoes translational motions according to one direction (front and back) which correspond to driver's acceleration and deceleration. The overall system's design allows having a simple linear model of the motion. Table 1 contains a nomenclature of different symbols.

\section{A. The linear motion platform:}

The motion base supports the cabin consisting of the seat, the vehicle board and the driver. Because the rotations of the seat are slow and low in amplitude, its induced inertia is negligible comparing to the total mass of the cabin's set. The linear motion of the cabin's set is made thanks to a ball screw/nut transmission mechanism driven by a DC actuator. The technological design was made in order to reduce, even eliminate, mechanical flaws such as backlash, mechanical play, static and dynamic friction, and to be able to design good quality acceleration and jerk based controllers. The following equations describe the systems components.

The actuator's electric equation is:

$u-e=R_{1}+L_{1} \frac{d i}{d t}$

The mechanical equation of the actuator pulling the cabin is:

$T_{a 1}=J_{a 1} \frac{d \omega_{a 1}}{d t}+f_{a 1} \omega_{a 1}+\frac{T_{a l 1}}{N_{1}}$
It is a standard to relate the torque $T_{a 1}$ developed in the rotor in terms of the armature current $i$ and a constant $K_{t 1}$, and to express the generated voltage as a results of the shaft rotational velocity $\omega_{a 1}$ and the back emf $e$, that is:

$$
\begin{aligned}
& T_{a 1}=K_{t 1} i \\
& e=K_{e 1} \omega_{a 1}
\end{aligned}
$$

We have now two more components: the ball screw-nut transmission mechanism and the cabin's set. The last is considered as a whole having a mass $M$ sliding on a mechanical guide-way under an external applied force $F_{x 1}$. The guide-way induces friction during motion. The entire cabin set slides according to the $\vec{x}$ axis. The governing equation is:

$M \frac{d \dot{x}}{d t}+f_{x 1} \dot{x}=F_{x 1}$

The balls screw/nut pulling mechanism is driven by the external torque $T_{s 1}$, indeed:

$T_{s 1}=J_{s 1} \frac{d \omega_{s 1}}{d t}+f_{s 1} \omega_{s 1}+T_{s l 1}$

Now it is to link the three systems. First, linking the pulling mechanism to the cabin's set is made through the variables $T_{s l 1}$ and $F_{x 1}$. in fact the load torque $T_{s l 1}$ is transformed through the linkage to the axial force $F_{x 1}$ by the following equation:

$T_{s l 1}=\frac{p_{1}}{2 \pi \eta} F_{x 1}$

Equation (5) can be written as:

$T_{s 1}=J_{s 1} \frac{d \omega_{s 1}}{d t}+f_{s 1} \omega_{s 1}+\frac{p_{1}}{2 \pi \eta}\left(M \frac{d \dot{x}}{d t}+f_{x 1} \dot{x}\right)$

Linking the pulling ball screw/nut mechanism to the actuator is made through the variables $T_{s 1}$ and $T_{s l 1}$.

Indeed, the actuator load torque is in fact the applied screw torque and thus $T_{s 1}=T_{a l 1}$ and equation (2) becomes:

$T_{a 1}=J_{a 1} \frac{d \omega_{a 1}}{d t}+f_{a 1} \omega_{a 1}+$

$\frac{1}{N_{1}}\left[J_{s 1} \frac{d \omega_{s 1}}{d t}+f_{s 1} \omega_{s 1}+\frac{p_{1}}{2 \pi \eta}\left(M \frac{d \dot{x}}{d t}+f_{x 1} \dot{x}\right)\right]$

Now we can work this equation either in the cabin Cartesian space $x$ or the actuator joint space, this can be done simply by the equation linking the rotational speed to the Cartesian's one:

$\dot{x}=\frac{p_{1}}{2 \pi} \omega_{s 1}$

and the one linking the actuator speed to the screw pulling system's one through the reduction factor $N_{1}$, that is: $\omega_{s 1}=\omega_{a 1} / N_{1}$

Finally we obtain the following equation considering the cabin's motion space: 


$$
\begin{aligned}
& K_{t 1} i=\underbrace{\left(\frac{2 \pi N_{1}}{p_{1}} J_{a 1}+\frac{2 \pi}{p_{1} N_{1}} J_{s 1}+\frac{p_{1}}{2 \pi \eta N_{1}} M\right)}_{J_{1}} \frac{d \dot{x}}{d t}+ \\
& \underbrace{\left(\frac{2 \pi N_{1}}{p_{1}} f_{a 1}+\frac{2 \pi}{p_{1} N_{1}} f_{s 1}+\frac{p_{1}}{2 \pi \eta N_{1}} f_{x 1}\right)}_{f_{1}} \dot{x}
\end{aligned}
$$

Since:

$u=R_{1} i+L_{1} \frac{d i}{d t}+\frac{2 \pi N_{1} K_{e 1}}{p_{1}} \dot{x}$

and using the well-known Laplace Transform, we can obtain the transfer functions between the cabin's position $X(s)$ and the voltage command signal $U(s)$ :

$$
\frac{X}{U}=\frac{1}{s} \frac{K_{t 1}}{\left(J_{1} s+f_{1}\right)\left(L_{1} s+R_{1}\right)+\frac{2 \pi N_{1}}{p_{1}} K_{e 1} K_{t 1}}
$$

\section{B. The rotating seat model}

As previously stated, the driver seat can perform two kinds of small rotational motions: the rotation of the only seat's back and the entire seat rotation. A single actuator with a manual switch performs either the first or the second functionality but not both at time. This motion can be coupled to the linear one giving five possible combinations for experimental investigations of motion feedback strategy during vehicle acceleration and braking using a modeling approach similar to that of cabin supporting platform, that is: the seat set can be split into three subsystems: the actuator set, the balls screw/nut transmission mechanism, and the seat set (including driver). In the actuator level, the equations remain the same, and the parameters are taken according to the actuator and the reduction factor. The balls screw/nut pulling system is also similarly modeled taking. The load torque at the nut/screw interaction level $T_{s / 2}$ generates an axial force $F_{x 2}$, such that, as for equation (7):

$T_{s l 2}=\frac{p_{2}}{2 \pi \eta} F_{x 2}$

At the seat level, things appear to be not simple. We are intersected in achieving small rotation angles of either the seat or the seat back, then the center of gravity and the inertia parameters are difficult to obtain because the driver can be anyone and he/she can take different driving postures. Then, the forces inducing rotations of either the seat or the back are: the torque induced by gravity, the one induced by the screw/nut, we have:

$m_{t} g \rho \sin (\theta+\phi)+F_{x 2} l=J_{t 2} \ddot{\theta}+$

$m_{t}\left[\ddot{x} \rho \cos (\theta+\phi)+\ddot{\theta} \rho^{2}\right]$

Equation (15) is expressed in function of $F_{x 2}$ such:

$$
\begin{aligned}
& F_{x 2}=\frac{J_{t 2}}{l} \ddot{\theta}+\frac{m_{t}}{l}\left[\ddot{\theta} \rho^{2}-g \rho \sin (\theta+\phi)\right. \\
& +\underbrace{\ddot{x} \rho \cos (\theta+\phi)}_{K(\theta, \ddot{x})}]
\end{aligned}
$$

This equation is nonlinear, thus we gather the nonlinear terms into the function $K(\theta, \ddot{x})$. Since the screw speed relates the induced linear motion by: $\omega_{s 2}=\frac{2 \pi}{p_{2}} \dot{x}$ when $x$ covers an arc around $\vec{y}$ by a ray of $l$ (figure 2), then $x$ related $\theta$ by $x=l \theta \Rightarrow \dot{x}=l \dot{\theta}$, then $\omega_{s 2}=\frac{2 \pi}{p_{2}} l \dot{\theta}$.

Now, replacing each item, in a way similar to the motion platform, modeling gives:

$$
\begin{aligned}
& K_{t 2} i=\left(\frac{2 \pi l N_{2}}{p_{2}} J_{a 2}+\frac{2 \pi l}{p_{2} N_{2}} J_{s 2}+\frac{p_{2}}{2 \pi \eta l N_{2}}\left(J_{t 2}+m_{t} \rho^{2}\right)\right) \ddot{\theta} \\
& +\left(\frac{2 \pi I N_{2}}{p_{2}} f_{a 2}+\frac{2 \pi l}{p_{2} N_{2}} f_{s 2}\right) \dot{\theta}-\frac{p_{2}}{2 \pi \eta l N_{2}} m_{t} g \rho \sin (\phi+\theta) \\
& +\frac{p_{2}}{2 \pi \eta l N_{2}} m_{t} \mathrm{~K}(\theta, \ddot{x})
\end{aligned}
$$

The obtained equations are nonlinear. Preliminary considerations concern the seat's rotation angle. Because we want to feedback vehicle motion during acceleration and braking situation in a reduced space, we will be using tilting and washout techniques in the same way as in flight simulators. However, feeding back maintained acceleration requires tilting the seat in a way such that the gravity will act on the operator's otoliths. Nevertheless the tilting angle and speed must lie under a certain threshold otherwise the operator is aware of the seat tilting and illusion falls. Consequently, the tilting angle must be kept small $\left(\theta<10^{\circ}\right)$.

In this case, one can make the well known approximations:

$\sin \theta \approx \theta$ and $\cos \theta \approx 1$, then:

$K(\theta, \ddot{x})=\rho \ddot{x} \cos (\phi) \theta$

If there is no motion then $F_{x 2}=0, \ddot{x}=0$, the overall equation (16) is linearized in the neighborhood of $-10<\theta<10$; otherwise the equation is still nonlinear because $F_{x 2}$ varies according to time and the nonlinear term

$\ddot{x} . \theta$ can be linearized by dynamic state feedback approach (other appropriate approaches can be developed). 


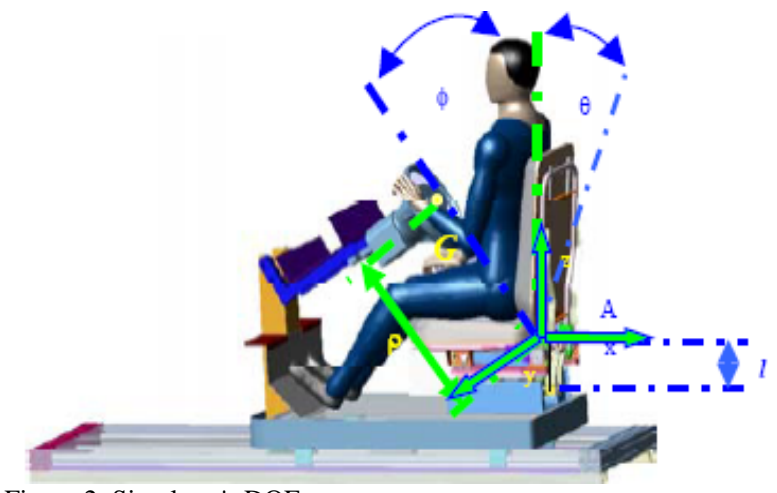

Figure 2: Simulator's DOF

\section{WASHOUT ALGORITHMS}

In order to give to the driver the illusion of feeling the inertial effects of the simulated vehicle (acceleration and deceleration transitions), the platform is powered by a washout algorithm. A washout algorithm aims to feedback transitory accelerations, considering the kinematics and the mechanical/dynamic limitations of the platform (workspace, robustness, bandwidth, etc.).

Several algorithm were been proposed to performing washout such classical method, optimal and adaptive one.

\section{A. Classical washout}

Our simulator is a 2 DOF one, but in reality we can't exploit the rotation of the seat for the tilt-coordination technique (only the seat rotates with respect to the longitudinal platform, and the last one is fixed in the world frame). Thus we can restitute just the longitudinal acceleration by passing it into a high-pass filter to extract the transitory component. The selection of the high-pass filter parameters takes place according to the maximum platform dimension [6,9]. After having filtered the acceleration, the signal produced is filtered by another high-pass filter to bring the platform to her neutral position. The resulting signal is integrated twice in order to obtain the desired position profile [2]. This is commonly referred to as "washout". It is important to emphasize that the time constant of the second filter must be chosen as to allow the platform returning to its initial position without crossing the vestibular system's movement perception threshold. If not, the subject will perceive a contradiction between the visual and the vestibular stimulation.

\section{B. Adaptive washout}

Adaptive algorithms are more flexible and intelligent comparing to the classic one [10]. It uses a set of filter to whose coefficients are systematically varied in real time to minimize a cost function. Although various schemes have been proposed and implemented for flight simulator motion cueing $[10,11,12]$. Its adaptation to our simulator was possible without great modification. Input to this algorithm is the filtered longitudinal acceleration (variation component) of vehicle. A cost function is minimized to optimize the acceleration error between simulator and vehicle with applying constraints (position and velocity) to the simulator motion.

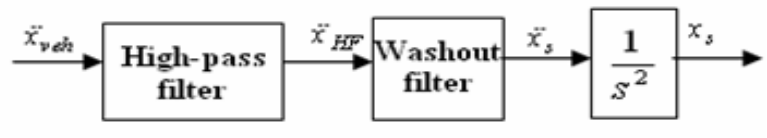

Classical washout

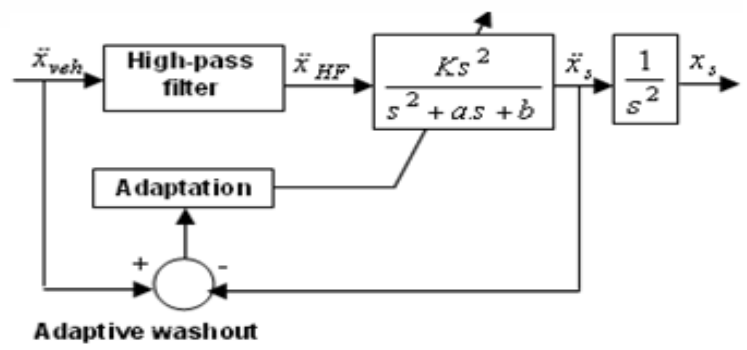

Figure 3: adaptive and classic filter synoptic

Position to filtered acceleration transfer function is giving by :

$\frac{x_{s}}{\ddot{x}_{H F}}=\frac{K}{s^{2}+a s+b}$

That is expressed in differential equation form by:

$\ddot{x}_{s}=K \ddot{x}_{H F}-a \dot{x}-b x$

The cost function to be minimized has the following form[10]:

$J=\frac{1}{2}\left[w_{a}\left(\ddot{x}_{H F}-\ddot{x}_{s}\right)^{2}+w_{v} \dot{x}_{s}^{2}+w_{p} x_{s}^{2}\right]$

Optimization is processed by the steepest descent method, that:

$\dot{K}=-\gamma \frac{\partial J}{\partial K}$

And the adaptive filter is obtained by resolving one first order and one second order differential equations of form:

$\dot{K}=\gamma\left[w_{a}\left(\dddot{x}_{H F}-\ddot{x}_{s}\right) \frac{\partial \ddot{x}_{s}}{\partial K}-w_{v} \dot{x}_{s} \frac{\partial \dot{x}_{s}}{\partial K}-w_{p} x_{s} \frac{\partial x_{s}}{\partial K}\right]$

and

$\frac{\partial \ddot{x}_{s}}{\partial K}=\ddot{x}_{H F}-a \frac{\partial \dot{x}_{s}}{\partial K}-b \frac{\partial x_{s}}{\partial K}$

\section{Simulation}

We had only simulated the longitudinal motion (position, velocity and acceleration) of the proposed simulator figure (4). Simulation is carried out using Matlab/Simulink Software. For comparison, we have taken the same washout filter parameters for classical algorithm and the one adaptive, with one exception that gain is variable for the adaptive scheme. For this, the different weightings are adjusted to give the desired motion in response of a one braking maneuver. Results are shown in figures (5).

The two first figures is a comparison between the 
acceleration and position cueing using classical and adaptive algorithms respectively. No noticeable difference between the two algorithms. The adaptive algorithm is little better in coordinated motion which is not the case herein. In literature [10,11,13], authors haven't explicitly shown how the comparison was made up between the two algorithms, and which parameters have been taken to do it.

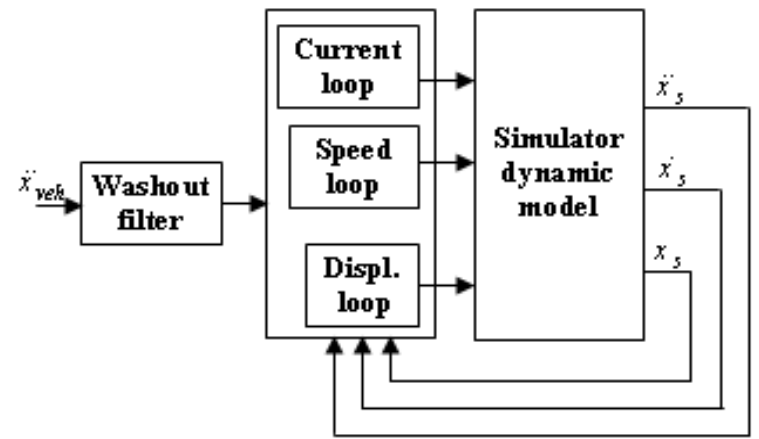

Figure 4: Simulator simulation diagram
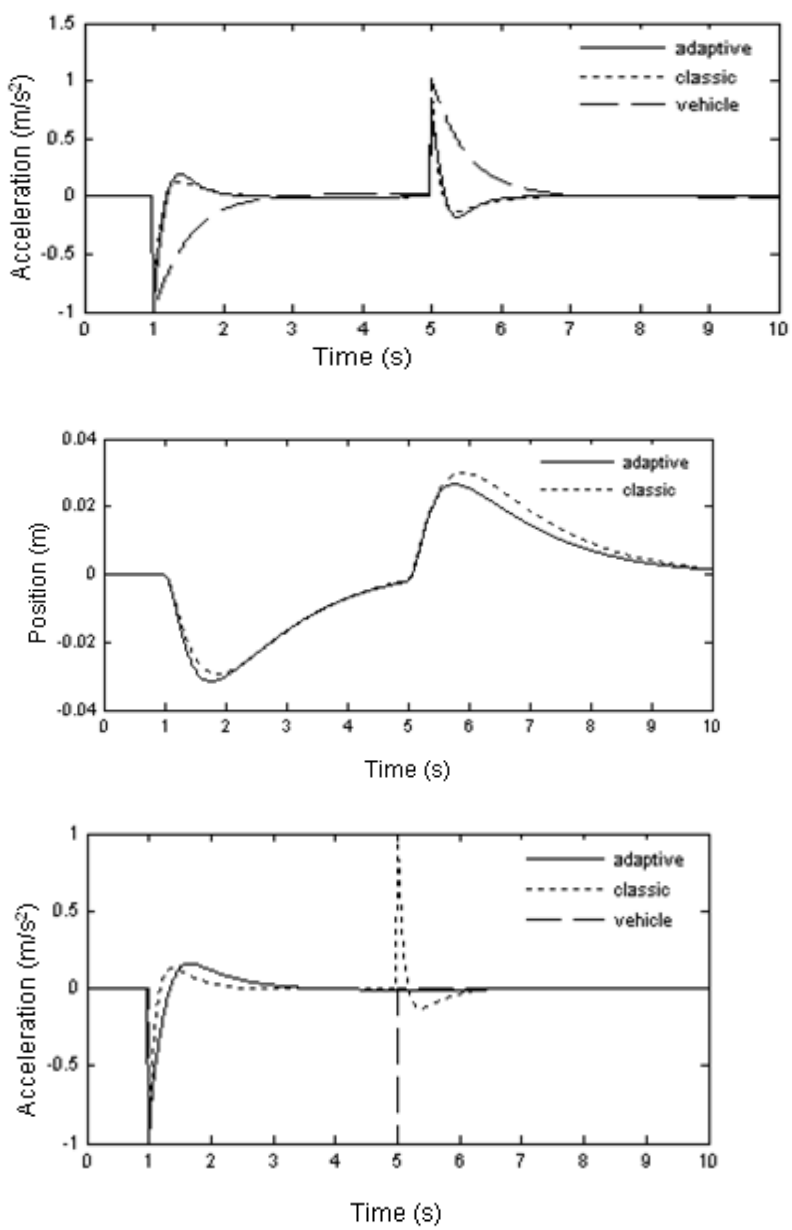

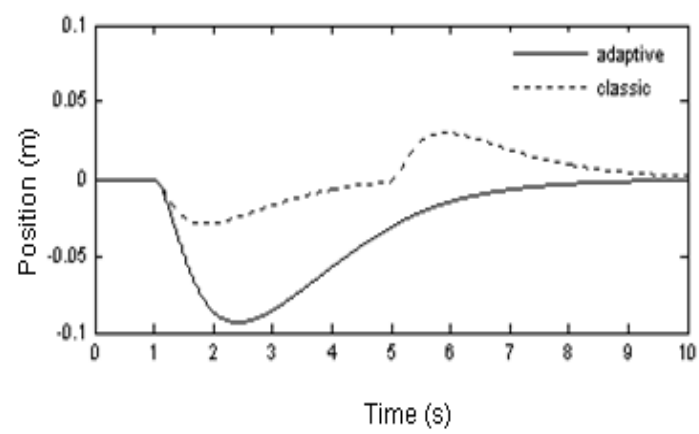

Figure 5: results of simulation of a braking maneuver using classical and adaptive algorithm

The two last figures show the acceleration and position of adaptive and classical washout algorithms respectively. For adaptive scheme, the high-pass filter was suppressed to remove the backlash, which is a characteristic of linear high-pass filters [6].

Figure (6) show a simulation of the simulator's position in a response of a reference position produced by the previous classical washout filter. The simulator has a good characteristic of tracking.

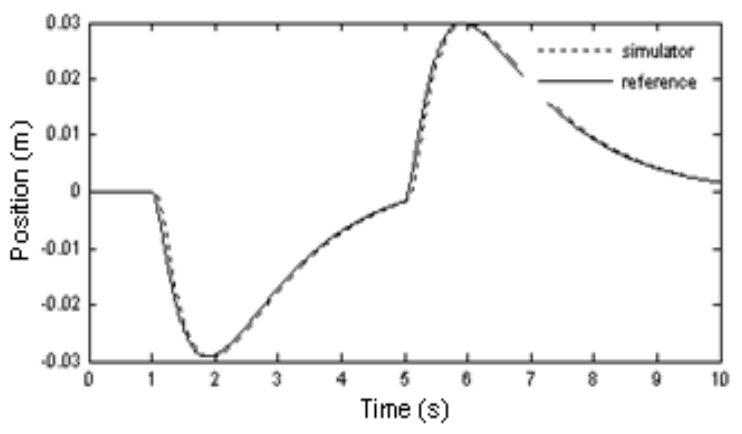

Figure 6: Simulator' position in response at a classical washout reference of position

\section{CONCLUSION}

The aim of this article is to experiment two kinds of washout algorithms, and to conclude which is more adapted for our small driving simulator. Classical washout algorithm is easiest to adjust, but it produces significant false cues due to the linear form of the filter.

Adaptive algorithm is more intelligent in which the gain of the filter varied according to the vehicle input acceleration. But tuning parameters is difficult and more subjective comparing to the classical algorithm. Nevertheless, because of the complexity of the adaptive scheme, no stability analysis could be performed. Simulations have shown that the proposed algorithm is stable with some difference in time convergence according to the adaptation step of the steepest descent method.

In this study, we can conclude that for small and low cost simulator, classical washout filter provide sufficient response if the corresponding parameter are well selected. Adaptive filter could be more interest in the case where the 
platform can tilt the cabin of driver to provide the sustained component of acceleration.

Due to the limited space in the present paper, we can't provide more results of other maneuvers simulation. In this time, experimentations and psychophysics validations are carrying out at INRETS of Arcueil (the National Institute of transportation researches and Security) to test the behavior of drivers and validate the system.

Other washout algorithms will be implemented at the simulator and tested to make a general comparison in order to adopt one to be implemented on the driver simulator.

\section{REFERENCES}

[1] G. Reymond, A. Kemeny, J. Droulez, A. Berthoz, "Role of Lateral Acceleration in Curve Driving: Driver Model and Experiments on a Real Vehicle and a Driving Simulator”, Human Factors, Vol. 43, No. 3, pp. 483-495.

[2] G. Reymond, A. Kemeny , J. Droulez, A. Berthoz, "Contribution of motion platform to kinesthetic restitution in a driving simulator", DSC2000, Paris, France, pp 33-55, July 1999.

[3] A. Kemeny "Simulation et perception du mouvement", Driving Simulation Conference, DSC’99, pp33-p55, Paris, France, July 1999.

[4] A. Kheddar, Ph. Garrec, "Architectures des plateformes mobiles pour simulateurs de conduite automobile”, CRIIF - 2002.

[5] G. Reymond, A.Kemeny, "Motion cueing in the Renault driving simulator”, Vehicule System Dynamic, Vol. 34, N 4, October 2000, pp. 249-259.

[6] H. Mohellebi, S. Espié, A. Arioui, A. Amouri and A. Kheddar, "Low cost motion platform for driving simulator" 5th International Conference on Machine Automation, ICMA'04, Osaka, Japan, 24-26 November 2004

[7] P-R. Grant, L-D. Reid, "Motion washout filter tuning: rules and requirements”, Journal of Aircraft, Vol. 34, N² 2, March-April 1997, pp. 145-151.

[8] S-F. Schmidt, B. Conrad, "Motion drive signals for piloted flight simulators” NASA CR-1601, May 1970.

[9] E-L. Groen, M-V. Clari, R. Hosman, "Psychophysical thresholds associated with the simulation of linear acceleration", AIAA 20004294.

[10] M-A. Nahon, L-D. Reid, "Simulator motion-drive algorithms: a designer's perspective”, Journal of Guidance, Vol. 15, Nº2, MarchApril 1992,pp. 356-362.

[11] R-V. Parrish, J-E. Dieudonne, R-L. Bowles, D-J. Martin, "Coordinated adaptive washout for motion simulators", Journal of Aircraft, Vol. 12, Nº 1, January 1975, pp. 44-50

[12] D. Ariel, R. Sivan, "False cue reduction in moving flight simulators", IEEE Transactions on Systems, Man and Cybernetic, Vol. 14, $\mathrm{N}^{\circ} 4$, July-August 1984, pp. 665-671.

[13] L-D. Reid, M-A. Nahon, "Response of airline pilots to variations in flight simulator motion algorithms", Journal of Aircraft, Vol. 25, $\mathrm{N}^{\circ}$ 7, July 1988, pp. 639-646.

\begin{tabular}{|c|c|c|}
\hline Symbol & Quartity & Unity \\
\hline $2, e$ & $\begin{array}{l}\text { Amature applied and back } \\
\text { electromotrioe voltage }\end{array}$ & Volt \\
\hline$R I$ & Amature resitance and inductance & $\begin{array}{l}\text { Olm, } \\
\text { Herry }\end{array}$ \\
\hline$i$ & Amature cumert & Anpere \\
\hline$T$ & Torque & N.m \\
\hline$J$ & Rotational mertia & $\mathrm{Kg} \cdot \mathrm{m} 2$ \\
\hline$f$ & Friction & \\
\hline$N$ & Reduction factor & \\
\hline $\mathrm{K}, \mathrm{K}$ & Electrical constart & \\
\hline in & Cabin's and estmated operator's mass & $\mathrm{Kz}$ \\
\hline$m_{i}$ & Seat's and etimated operator's mass & \\
\hline$F$ & $\begin{array}{l}\text { Axial force generated at the rutlscrew } \\
\text { interaction brel }\end{array}$ & $\mathrm{H}$ \\
\hline$p$ & Screw thread & $\mathrm{m}$ \\
\hline$\Pi$ & Screw's yield & \\
\hline$\frac{1}{L}$ & screw/rout axis length & $\mathrm{m}$ \\
\hline$\rho$ & $\begin{array}{l}\text { Estimated distance between grarity } \\
\text { certre and rotation axe }\end{array}$ & $\mathrm{m}$ \\
\hline$d$ & screw/rout axis diameter & $\mathrm{m}$ \\
\hline$g$ & Gravity force & $\mathrm{H}$ \\
\hline a & Rotational speed & radiansis \\
\hline$\frac{d x}{d y}$ & $\begin{array}{l}\text { derivative of function } x \text { acoording to } \\
\text { variable } y\end{array}$ & \\
\hline$\dot{x}, \ddot{x}$ & first and second derivative of $x$ & \\
\hline$\theta$ & Seat rotation angle & radians \\
\hline$K_{a, b}$ & Washout filter's parameters & \\
\hline$t$ & time & $\begin{array}{l}\text { Seconds } \\
\text { (s) }\end{array}$ \\
\hline \multicolumn{3}{|l|}{ Subscript } \\
\hline 1,2 & $\begin{array}{l}\text { refer to the platform and seat actuator's } \\
\text { variables }\end{array}$ & \\
\hline$a, s$ & $\begin{array}{l}\text { refer to actuator and screw/rnt's } \\
\text { variables }\end{array}$ & \\
\hline al, $s !$ & $\begin{array}{l}\text { refer to actuator bad and screwirnt's } \\
\text { load variables }\end{array}$ & \\
\hline$s$ & refer to sirvulato's variables & \\
\hline$x$ & $\begin{array}{l}\text { refer to longitudinal force generated by } \\
\text { the bad torque }\end{array}$ & \\
\hline$H F$ & $\begin{array}{l}\text { refer to the high-frequency component of } \\
\text { acceleration }\end{array}$ & \\
\hline
\end{tabular}

Table 1: Nomenclature and symbols 